\title{
MHD. EIN ALS DEMONSTRATIVPRONOMEN.
}

Ein gar nicht seltener gebrauch von mhd. ein, auf den ich in meinen mhd. ubungen schon seit vielen jahren aufmerksam zu machen pflege, scheint bisher ganz unbekannt geblieben zu sein, da in den wörterbuchern nichts dartber angemerkt ist ${ }^{1}$ ) und selbst männer wie Lachmann und W. Wackernagel an einigen stellen durch conjectur den ihnen unverständlichen gebrauch wegschaffen zu durfen geglaubt haben.

Es kann nämlich mhd. ein in der verbindung mit einem substantiv gebraucht werden in stark hervorhebender bedeutung, wo wir zum mindesten den bestimmten artikel brauchen mussen, während in den meisten fällen die nhd. tbersetzung durch 'jener' am angemessensten erscheint. Dieser gebrauch von ein ist bisher immer nur vom standpunkte unseres nhd. unbestimmten artikels aus betrachtet und beurteilt worden. Mit diesem aber darf er nicht vermengt werden, wenngleich man in manchen weniger ausgeprägten făllen wol zuweilen mit unserem unbestimmten artikel auskommt. Man wird vielmehr dabei direct an das zahlwort ein anknupfen mulssen, indem die bedeutung 'einzig' Uberfuhrt zum begriff des bestimmten, eben durch sein alleinstehen bekannten und so mhd. ein man gradezu heissen kann 'jener Eine, hinlänglich bekannte mann', 'jener mann', 'der mann'. Viel ähnliches hat mit diesem gebrauche die anwendung des lat. ille zur hervorhebung von etwas bekanntem, bestimmtem.

Im nhd. ist dieser hervorhebende gebrauch von ein nicht mehr ublich. Höchstens könnte in dem hervorhebenden ein vor eigennamen (ein Lessing, ein Gioethe, ein Alexander), das

1) Was Grimm, Wb. III, 130-133 von hierhergehörigen beispielen hat, entbehrt sebr des zusammenhanges. 
dem gebrauch des lat. ille (Cicero ille etc.) entspricht, noch ein abkömmling des altdeutschen gebrauchs vorhanden sein. ${ }^{1}$ )

Gänzlich erstarrt und unverstanden haben wir das alte ein noch im kanzleistile, in der anrede an behörden u. dgl., aber nur, wenn damit noch ein attributives adjectiv verbunden ist: ein hohes ministerium, eine hohe landesregierung, ein löblicher magistrat etc. Hier sagt uns unser jetziges sprachgefubl, dass da eigentlich nur der bestimmte artikel stehen durfte; jedoch dämmert uns noch, dass das ein hier mehr wie der bestimmte artikel sagen solle und eine besondere respectbezeugung sei. Und so ist es auch. Die behörde soll dadurch als die Eine, einzig in betracht kommende, die jedermann kennt, bezeichnet werden. Im älteren neulochdeutsch des 16. jh.'s war das respectvolle dieses ein noch deutlich empfunden, noch im lebendigen redegebrauche und noch nicht bloss auf den kanzleischreibstil verwiesen wie bei uns. So z. b., wenn in dem volksliede vom kranzsingen (Uhland, Volksl. s. 10) der auftretende sănger begr(dsst $\left.{ }^{2}\right)$ : ein erbern weisen rat, ein erbern rat nicht alleine, darzu ein ganze gemeine. ein erbern rat hab ich wol zu grüssen macht, gott grüss mir ein ganze nachbarschaft. Nachdem er diesen erhabenen körperschaften das respectvolle ein gewidmet hat, wendet er sich mit dem bestimmten artikel an das junkfrewlin zart, dio den kranz gemacht hat. - Auch ohne attribut wird ein in dieser weise im 16. jh. gebraucht, z. b. in einem triumphliede der Schweizer uber die landsknechte (Uhland 8.442) wend sie me an ein eidgenoschaft, drü paner müssends machen; oder aus Hans Sachs: dass heisset uns ein rhat dir klagen; ebend.: was hat ein rhat

1) Unser beutiges sprachgefuhl wird allerdings geneigt sein, fällo wie 'ein Lessing' etc. als ubergang des eigennamens in ein appellativum sich zu erklären, also = 'ein mann wie Lessing'. Doch dürfte darin kaum der ursprung des gebrauchs zu sehen sein, da ein Lessing wol zunächst 'jener bekannte, berühmte Lessing' bedeutete und also auf das altdeutsche ein zurlickweist. Grimm (Wb. III, 132) bemerkt, statt 'das kann ein Lessing nicht geschrieben, ein Goethe nicht gedichtet haben' sage man besser 'Lessing, Guethe' ohne artikel; aus welcher bemerkung Grimm's erhellt, dass anch dieser gebrauch des ein schon fremdartig and veraltet erscheint.

$11 \mathrm{II}$.

2) Dieses und die drei folgenden beispiele nach Grimm, Wb. 
beschliessen than? u. a. In all diesen beispielen durften wir durchaus nur den bestimmten artikel statt des ein in anwendung bringen.

Aber auch vor anderen substantiven war das hervorhebende, demonstrative ein im 16. jh. noch gebrãuchlich. Ein sehr lehrreiches beispiel, an welchem mir diese bedeutung von ein uberhaupt erst klar geworden ist, bietet Luthers uber-

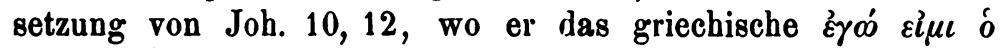

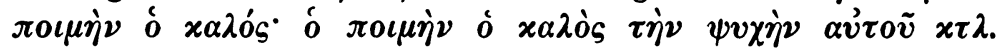
ubersetzt: 'ich bin ein guter hirte, ein guter hirte lässt sein leben fur die schafe' und ebenso nochmals v. 14 'ein guter

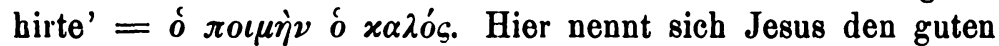
hirten $\varkappa \alpha \tau^{\prime}$ ' $\xi o \chi \eta^{\prime} v$, den alleinigen, bekannten guten hirten. Es ist sicher, dass ein fur Luthers sprachgefuhl diesem sinne besser entsprach als der, welches ihm ja sonst dem griech.o entsprechend am nächsten gelegen hätte: pastor ille bonus wüde man Luthers 'ein guter hirte' lateinisch geben mulssen. Man sieht auch hier, mit welcher sorgfalt Luther, ohne sich ängstlich an den buchstaben zu halten, den sinn des originals scharf auszuprägen trachtete. Unserem jetzigen sprachgefuble bleibt freilich diese sprachliche feinheit Luthers verschlossen und ich erinnere mich seiner zeit in der religionssstunde bei dieser stelle gehört zu haben, Luther habe hier fälschlich ó durch den unbestimnten artikel ubersetzt - eine stumperei, welche dreimal hintereinander $z u$ begehen Luther doch nicht fähig gewesen sein würde.

Der gebrauch dieses ein lässt sich bei Luther noch vielfach aufzeigen; am blindigsten beweisen die bibelstellen, weil das griech. original in diesem falle stets den bestimmten artikel hat. So z. b. Joh. 1, 21 die frage an Johannes den tăufer: i $\pi \rho \circ \varphi \eta \dot{\tau} \eta \varsigma \varepsilon \varepsilon \bar{l} \sigma \dot{v}$; (d. b. jener bekannte, bestimmte, von Moses geweissagte prophet) lubersetzt Luther: 'bist du ein prophet?', für seine zeit ebenso richtig, wie für uns unverständlich. Ferner

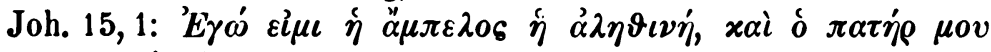

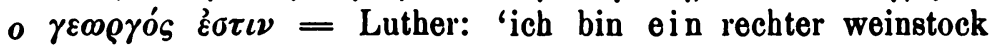
und mein vater ein weingärtner'.

Ein anderes hubsches beispiel des bestimmten gebrauches von ein aus dem 16. jl. habe ich vor vielen jahren in dem volksbuche von Fierabras (Simmern 1533) gelesen, mir aber 
die stelle nicht ausgeschrieben, so dass ich nur citieren kann worauf es aukommt. Es heisst da von einem helden: 'er war ein vater des $x$ ', wo wir dem gewöhnlichen laufe der dinge entsprechend doch nur 'der vater' fur angemessen balten können.

Im mhd. war dieser hervorhebende gebrauch des ein ganz allgemcin und ich gebe nun dazu liber denselben durch beispiele zu belegen. Meine beispiele sind ohne systematische sammlung durch gelegentliches notieren zusammengebracht; doch werden sie genugen, um den gebrauch des demonstrativen ein für die blutezeit der mhd. dichtung als durchaus normal nachzoweisen und ihn gegen fernere missdeutungen sicher zu stellen. Aus dem älteren mhd. habe ich nur zwei stellen zur hand: weiteres nachsuchen in der litteratur des 12. jh.'s würde gewiss noch manchen beleg ergeben. Vor dem 12. jh. (ahd.) sind mir allerdings beweisende stellen zur zeit nicht bekannt.

Ich stelle die zwei ältesten stellen voran:

Vorauer Alexander, Diemer 192, 2 = Kinzel 315 (S. ändert stark)

Alexander begunde dô streichen ein ros daz nie nichein man begunde weichen.

Es ist vorher ausfuhrlich von der unbändigkeit des Bucival erzählt und eben berichtet worden, wie beim herankommen Alexanders das ross wie umgewandelt war; es kann also die stelle nur heissen: 'Alexander begann da zu streicheln jenes (vielbesprochene) ross, welches vorher noch niemand zu bändigen unternommen hatte'.

\section{Rolantslied $308_{17}$ (Grimm)}

Des gerte di edele herzoginne

aines richen chuniges barn.

Es ist dio rede von Gertrud, gemahlin herzog Heinrichs von Baiern, tochter des deutschen königs Lothar. Da der in Baiern lebende dichter doch bei seinem publicum voraussetzen musste, dass alle die herkunft der herzogin kannten, so musste die erwähnung des kōnigs als eines rîchen kuniges allerdings schief erscheinen 1), wenn nicht ein ein rîcher chunig hier hiesse:

1) E. Schröder zs. fda. 27, 81 nimmt denn auch an dem 'unbestimmten artikel' anstoss. 
'der allbekannte mächtige könig, unser könig Lothar'. Es ist also vom dichter eine noch stärkere bervorhebung als mit dem einfachen bestimmten artikel beabsichtigt.

Es folgen nun stellen aus dichtern der bluteperiode.

Wolfram P. 442 28. 29. Parzival ist der frischen spur Cundrîens nachgefolgt (dô kêrter ûf die niuwen slâ 442 26 ); darauf heisst es:

daz ungeverte im undervienc

eine slâ dier het erkorn

Wackernagel conjicierte hierzu gegen alle hss. jene slâ, wie Lachmann in den varianten angibt; jedenfalls wäre im nbd. mindestens der bestimmte artikel zu setzen, wis auch Simrock tut: 'dass bald im dichten waldgehege die spur verschwand, die er erkorn'.

Ganz ebenso P. $553_{11}$

er kôs ein burc, diers âbents sach,

dô im diu âventiure geschach.

Der burg sind vorher $\left(534_{20}\right)$ schon zehn verse gewidmet; es heisst also ein burc hier 'jene schon bekannte burg'. Die conjectur jene wäre hier ebenso angebracht, wie bei der vorigen stelle. Simrock ubersetzt richtig 'da sah er bald die veste wider, die er tags zuvor gesehn'.')

Hartman, Armer Heinr. 1060 hat Wackernagel im lesebuch (V. aufl. 1873) in der stelle

er hete brâht eine maget,

die er in gewinnen hiez

in den text jene statt eine der hss. gesetzt. Die andern herausgeber folgen ihm darin nicht, wol weil man allenfalls mit dem nhd. unbestimmten artikel hier sich behelfen könnte. Aber das eine maget bezeichnet hier nicht irgend eine beliebige jungfrau, sondern eine bestimmte, in ilıren nälieren qualitäten schon vorher genau beschriebene. Wackernagel hat also die stelle richtig verstanden, wenngleich seine conjectur uberflussig ist, ebenso wie in obiger Parzivalstelle. Vgl. auch W.'s anm. zu dieser stelle in seiner grossen ausgabe (ed. Toischer, Basel 1885). In dieser ausgabe hat W. ausserdem in v. 1187 an in

1) [Auch in den mir nachträglich aufstossenden stellen P. 1356. $160_{17}$ dürfte ein durch den bestimmten artikel 211 geben sein.] 
einer kemenaten anstoss genomnen, aber statt des zuerst auch hier vermuteten jener schliesslich siner statt einer in den text gesetzt.

Wigalois (ed. Pfeiffer) $36_{10}$ ist von der jugend des Wigalois die rede; die sorgfalt, die von seiner mutter auf seine pflege verwant wurde, ist schon nachdrucklich hervorgehoben; dann heisst es:

\section{von rehte muos ez (das kind) sælic sîn ez zôch ein rîchiu künegîn unze zuo zwelf jâren.}

ein bedeutet hier die schon genugsam bekannte königin, seine mutter, ist also nhd. durch den bestimmten artikel zu ubersetzen.

Auch bei Walther v. d. Vogelw. findet sich der hervorhebende gebrauch des ein. So ist L. $43_{30}$ gar ein krône mit der höchste schmuck' zu übersetzen; - ferner L. $\mathbf{5 7}_{23}$ Minne diu hât einen site bedeutet 'Minne hat eine gewisse, eine bekannte gewohnbeit'. - Interessant ist die stelle L. 75. Die bessere uberlieferung in AC hat: lihte wirt mir einiu: sô ist mir sorgen buoz; die he. E stärker ändernd: vinde ich mîne, sô ist mir aller sorgen buoz. Die für uns vollkommen verständliche uberlieferung in AC beisst naturlich: 'vielleicht wird mir jene schon mehrerwähnte, jene eine bekannte, von der vorhin die rede war' etc. Lachmann setzte für das ihm unverstăndliche einiu mit einer contamination beider uberlieferungen mîniu (nach $\mathrm{E}$ ) in den text $\mathrm{AC}$, was nach ihm die meisten berausgeber beibehalten haben. Nur Wackernagel-Rieger in ibrer ausgabe (vgl. Einleit. 8. XXXIX) bessern im engern anschluss an AC einiu in eniu 'jene', dem sich Pfeiffer anschliesst: auch dieses naturlich eine böchst uberflussige änderung.

Im Wartburgkriege (ed. Simrock) $6_{7}$ steht ein Dürenge herre in beziehung auf den unmittelbar vorher genannten Herman von Dürengen lant; und $17_{1}$ beisst es von der anwesenden landgräfin: ein fürstin und ir frowen sint uns beiden al ze nâhe $b \hat{\imath}$ etc., was Simrock richtig ubersetzt: 'Die furstin und die frauen sind etc.'

Im Eraclius meint der neueste herausgeber Graef (QF. 50) s. 2 der einleitung, dass des bereits in v. 85 angefuhrten Perserkönigs Cosdroas später (v. 343 und 4488) in einer weise 
erwähnung geschehe, als ob noch niemals von ihm die rede gewesen sei, und rechnet dies dem dichter als vergesslichkeit an. Es beruht das bloss darauf, dass an beiden stellen Cosdroas als ein heiden angefubrt wird. Wenn man das richtig ubersetzt durch 'jener (schon erwähnte) heide', so verlieren beide stellen alles auffallende.

Eine besonders grosse anzahl von beispielen kann ich aus dem Nibelungenliede geben, da ich bei einer genaueren lectüre desselben reichlicher fur den gebrauch des demonstrativen ein gesammelt habe, während aus den ubrigen dichtern meine beispiele nur zufällig zusammengekommen sind. ${ }^{1}$ )

An verschiedenen bierhergehörigen stellen des Nibelungenliedes ist Lachmann zur conjectur geschritten, wenn die hss. ein in der beziehung auf etwas schon bestimmtes oder bekanntes haben. Diese stellen sind: $747_{1}$ an einem abende A BDIbd, an jeneme abende Lachm.; C ändert stärker, aber gewiss nicht, wie Liliencron s. 52 wähnt, wegen der angeblichen verderbnis einem; denn der demonstrative gebrauch des ein war $\mathbf{C}$ sehr wol geläufig, wie die folgenden stellen zeigen. - 479, in eime schiffe ABCd; in dem a, an dem DI, ime sch. Lachm. - $1710_{3}$ ab einem hase $\mathrm{Abd}$, von einem huse $\mathrm{B}$, az eime huse $\mathrm{C}$; abeme huse Lachm. - 214, af eime schilde alle hss., afem schilde Lachm. - 476 an einem morgen fruo

1) Allerdings scheint das Nibelungenlied diesen gebrauch des ein in besonders hohem grade zu haben. Jedoch würden sich wol in jedem mhd. schriftsteller eine reihe von beispielen bei genauer durchmusterung nachweisen lassen: besonders auch in den varianten, teils als lesarten einzelner hss., teils als vom kritischen herausgeber verworfene gesammtUberlieferung. Eine durchsicht der varianten des Meier Helmbrecht (ed. Haupt, Zs. fda. 4) ergab z. b. folgende fälle des ein in der bedeutung 'jener': $\mathbf{v} .73$ ener $\mathrm{H}$ (aupt) = einer $\mathrm{a}$, jener $\mathrm{b} ; 100$ enem $\mathrm{H}=$ ainem $\mathrm{a}$, jene $\mathrm{b} ; 963$ ener $\mathrm{H}=$ einer $\mathrm{a} b ; 1034$ enen $\mathrm{H}=$ enem $\mathrm{a}$, ainen $\mathrm{b} ; 1249$ enen $\mathrm{H}=$ einem a b. Man muss zugeben, dass Haupt an all diesen stellen mit recht ener in den text gesetzt hat, zumal es in einigen fällen besonders in a (vgl. auch 935. 1246) erhalten ist und in allen stellen rein locale, einem diser entgegengesetzte bedeutung hat ( $\mathrm{vgl}$. sogleich s. 525). Aber dass dem sprachgebrauche der schreiber die vertauschung von jener und einer nahe lag, geht daraus zum mindesten hervor. Vgl. z. b. auch die varianten zu Wolfram P. 511 $23.601_{25}$. 
A BDbd, vil fruo an cinem morgen $\mathrm{CI}$ a, an jenem $m$. fr. Lachm. Die beiden letzten stellen hat Lachmann nicht direct in den text gesetzt, sondern nur hinten unter den 'verbesserungen', aber sein sonst so strenger recensent (Germ. 7,196 ff.) ist grade mit diesen beiden conjecturen sehr einverstanden Und doch sind diese conjecturen nicht nur therflüssig, sondern Lachmann tritt an den beiden stellen, wo er das pron. jener einfubrt, sogar in widerspruch mit dem sonstigen sprachgebrauche des Nibelungenliedes. Denn jener in verbindung mit einem substantiv hat stets rein locale bedeutung, also = 'der dort' (vgl. $79_{2} .380_{1}, 477_{3}$ (C). 1636 $.1690_{2} .1823_{2}$ ); wo dagegen unser nhd. 'jener' nicht local ist, sondern 'der schon genannte' bedeutet, wird in Nibelungenliede immer ein gebraucht.1)

Als ein argument für seine strophenausscheidungen benutzt Lachmann das von ihm nicht verstandene ein 1493 ${ }_{1}$ : Vil hôhe anme swerte ein bouc er im dô bôt etc., nämlich 'jenen (schon str. 1490 genannten) bouc'. Lachmann bemerkt (zu den Nib. 1490. 1491) 'warum heisst es 1493 ein bouc, wenn er 1490 schon versprochen war'? In der recension $C$ ist hier der gleichbedeutende bestimmte artikel eingefuhrt: .. er im den bouc dô bôt.

Ebenso hat $\mathrm{C}$ in den zwei folgenden stellen geändert:

$653_{3}$ unze sie kômen zeiner bürge wît:

diu was geheizen Santen; dâ si krône truogen sît. AB $^{*}$

C: unze daz si kômen zer bürge wol bekant

rîche unde mære: diu was ze Santen genannt.

Die wendung von $\mathrm{C}$ zer bürge nol bekant ist nur eine paraphrase von zeiner bürge, welches eben heisst ' $z$ u jener (fruher schon genannten) burg'. Man darf also nicht wie Liliencron s. 48 in dem einer eine neueinfuhrung der burg sehen und daraus ein argument fur die liedertheorie hernehmen wollen.

1906. mit einem scharpfen swerte daz im gap Rüedegêr. Die bedeutung ist hier naturlich 'mit jenem schwerte, welches ihm Rudiger (1633) geschenkt hatte'. C liest mit dem schar-

1) Ausgenommen ist nur 491 zuo jenen tûsent recken, denn im plural, noch dazu neben dem zahlwort tâsent war ein nicht anwendbar. Es wäre darauf zu achten, ob auch andere quellen in ähnlicher weise sich des gebrauches von jener enthalten, wo ein angebracht ist. 
pfen swerle, weniger stark hervorhebend, aber fur den nhd. sprachgebrauch unanstössiger.

Umgekehrt steht $1889_{1}$ das hervorhebende ein in C, wăhrend die andern den bestimmten artikel habeu.

Vil late rief dó Dancwart eime degene $C$ zuo dem degene A B I.

Der bestimmte artikel, den Lachmann (anm. zur stelle) als unrichtig bezeichnet, wäre hier wol mit binblick auf das gleich folgende Hagene zu halten, denn auch eime ist hier keineswegs unbestimmter artikel, sondern emphatisch gebraucht.

An einigen weitern stellen steht das emphatische ein nur in A: $1493_{1}$ an einem swerte, $1696_{3}$ ein Guntheres man, $1700_{3}$ ein Elzelen wîp, $1918_{3}$ ein künec von Amelunge. Die tbrigen hss. haben hier sămmtlich den bestimmten artikel; Lachmann will uberall ändern. Da das hervorhebende ein durchaus ohne tadel ist, liegt natürlich fur den, der mit Lachmann $A$ als alleinige grundlage der kritik betrachtet, nicht der mindeste grund zur änderung vor, während andererseits der gegner von A diese ein nicht als einen beweis des ursprunglichen anzuerkennen braucht, da sie von jedem schreiber eingefuhrt werden konnten.

Ich zähle noch einige weitere stellen auf, welche beispiele unseres ein bieten, obwol sie der kritik nicht zum anstoss gereicht haben, da man sich gewöhnt hat, in ihnen einen freieren gebrauch des unbestimmten artikels zu sehen, wăhrend im nhd. der bestimmte artikel gesetzt werde. ${ }^{1}$ ) 896, ouch fuort er Balmungen ein ziere wâfen breit und ebenso 2287, ouch vorht er Balmunge, ein wafen starc genuoc, an beiden stellen hebt ein den Balmunc als 'jenes bekannte' schwert hervor, welches schon fruher öfter erwähnt ist. Ganz ebenso beurteilen sich die ein in appositionellen anfugungen an namen bekannter und schon im gedichte vielfach genannter persōnlichkeiten: ich fuhre nur beispielsweise an $992_{3}$ Vote ein edel wîp; $332_{3}$ Kriemhilde ein küneginne hêr u. a. $^{2}$ ) In all solchen stellen mussen

1) Vgl. besonders Rieger, Zur kritik der Nibelunge s. 61.

2) Ganz ebenso stellt sich nun die bekannte stelle 1416, Do kom der küene Volkèr, ein edel spilman, welche einfach mit 'der kühne spielnann' zu ubersetzen ist und nicht als 'neue einführung' Volkers geltend gemacht werden darf. 
wir in nhd. ein durch den bestimmten artikel geben, welchem aber das mhd. ein noch um einen stärkegrad voraus ist. Dass das mhd. ein in solchen wendungen bestimmende, demonstrative bedeutung hatte, seben wir auch an varianten einzelner hss. So z. b. $379_{4}$ Gunther, ein rîter küene unde balt $\mathrm{AB}$, der rîter $\mathrm{C}$; andere fälle von differenz der hss. sind $451_{1}$ ein schiffel $\mathrm{AB}$, daz sch. $\mathrm{C} ; \mathbf{2 2 3 4}_{3}$ ein starkez wâfen $\mathrm{AB}$, daz st. w. C; $205_{2}$ an ein ende $\mathrm{C}$, anz ende $\mathrm{AB}$. Es haben hier einzelne hss. die stärkere hervorhebung ein mit der etwas schwächeren vertauscht, so wie oft in mhd. hss. synonyme wechseln. Einen dem nhd. sprachgefuble entsprechenden vorgang hat man in solchen einsetzungen von der statt ein nicht zu erblicken.

Die vorstehenden beispiele werden, meine ich, gelehrt haben, dass man das mhd. ein durchaus nicht a priori vom standpunkte unseres unbestimmten artikels zu betrachten hat. Man wird demnach berechtigt sein, auch andere gebrauchsweisen des mhd. ein, welche unserem nhd. sprachgefuhle weniger schroff entgegentreten, vom standpunkte des hervorbebenden ein aug zu betrachten. So z. b. im mhd. sehr gebrăuchliche wendungen wie ze einem trate, ze einem herren han; ze einem man, zeiner vrouwen nemen, wo doch auch nichts unbestimmtes vorliegt und wir im nhd. sagen 'zum manne, zur frau nehmen' etc.

Auch das im mhd. ganz allgemeine ein vor vocativ, wovon Grimm, Wb. III, 135 beispiele anfuhrt, bekommt nun eine bessere beleuchtung, als vom unbestimmten artikel her; z. b. genade, ein küneginne! sinc, ein guldîn huon! lache, ein rôsevarwer munt! Hier setzen wir am besten ' $d u$ ' fur ein: 'lache, du rosiger mund'! ebenso schon in der sequenz aus Muri (Denkm. 42, ): ein lieht der cristenheit, Maria, aller magede ein lucerne: du licht der christenheit, Maria, du leuchte aller jungfrauen!

GIESSEN.

W. BRAUNE. 